TAIWANESE JOURNAL OF MATHEMATICS

Vol. 15, No. 5, pp. 2331-2336, October 2011

This paper is available online at http://tjm.math.ntu.edu.tw/index.php/TJM

\title{
CUBIC FORMAL POWER SERIES IN CHARACTERISTIC 2 WITH UNBOUNDED PARTIAL QUOTIENTS
}

\author{
K. Ayadi, M. Hbaib and F. Mahjoub*
}

\begin{abstract}
There is a theory of continued fractions for formal power series in $x^{-1}$ with coefficients in a field $\mathbb{F}_{q}$. This theory bears a close analogy with classical continued fractions for real numbers with formal power series playing the role of real numbers and the sum of the terms of non-negative degree in $x$ playing the role of the integral part. We give a family of cubic power series over $\mathbb{F}_{2}$ with unbounded partial quotients. To be more precise, let $f \in \mathbb{F}_{2}\left(\left(x^{-1}\right)\right)$ such that $f$ is not polynomial but $f^{3}$ is polynomial with degree $d \in 3 \mathbb{N}$, we prove that the continued fraction expansion of $f$ is unbounded.
\end{abstract}

\section{INTRODUCTION}

In [2], Khintchine conjectured that if $\mathrm{x}$ is a real algebraic number of degree $>2$ then $x$ has a continued fraction expansion whose the sequence of partial quotients is unbounded. Note that the answer to this conjecture is far from being tractable. However, more things are known in the case of algebraic formal series over a finite field $\mathbb{F}_{k}$ of characteristic $p$.

In 1976, Baum and Sweet gave, in [1], the first example of an algebraic formal series of degree 3 on $\mathbb{F}_{2}\left(\left(x^{-1}\right)\right)$ whose partial quotients have only a finite number of values as well as examples whose partial quotients have infinite values. In [4], Mills and Robbins continued the work of Baum and Sweet and they gave an example of formal series algebraic over $\mathbb{F}_{3}\left(\left(x^{-1}\right)\right)$ whose the sequence of partial quotients is unbounded and given explicitly. Moreover, Robbins gave a family of cubic formal power series with bounded partial quotients [5].

In this paper, we give a family of cubic formal power series with unbounded partial quotients. Namely, we prove that, if $f^{3}$ is polynomial with degree $d \in 3 \mathbb{N}$, then the continued fraction expansion of $f$ is unbounded.

Received February 4, 2009, accepted July 2, 2010.

Communicated by Wen-Ching, Winnie Li.

2010 Mathematics Subject Classification: 11A55, $11 \mathrm{R} 58$.

Key words and phrases: Finite fields, Formal power series, Continued fraction.

*Corresponding author. 


\section{Field of Formal SERIES}

Let $\mathbb{F}_{k}$ be a finite field with $k$ elements. We denote by $\mathbb{F}_{k}[x]$ the ring of polynomials with coefficients in $\mathbb{F}_{k}$ and $\mathbb{F}_{k}(x)$ its fractions field. Let $\mathbb{F}_{k}\left(\left(x^{-1}\right)\right)$ be the field of formal power series:

$$
\mathbb{F}_{k}\left(\left(x^{-1}\right)\right)=\left\{f=\sum_{i \geq n_{0}} f_{i} x^{-i} \quad: \quad n_{0} \in \mathbb{Z} \quad \text { and } \quad f_{i} \in \mathbb{F}_{k}\right\} .
$$

Let $f=\sum f_{i} x^{-i}$ be any formal power series, we define its polynomial part, denoted $[f]$, by $[f]:=\sum_{i \leq 0} f_{i} x^{-i}$. If $f \neq 0$, then the degree of $f$ is $\gamma(f)=\inf \left\{i: f_{i} \neq 0\right\}$ and $\gamma(0)=+\infty$. Thus, we define $|f|=k^{-\gamma(f)}$. Note that $|$.$| is a non archimedean$ absolute value over $\mathbb{F}_{k}\left(\left(x^{-1}\right)\right)$.

Now, similarly to the classical continued fractions for real numbers, we define the continued fractions for power series. Let us consider the set $M_{k}=\{f \in$ $\left.\mathbb{F}_{k}\left(\left(x^{-1}\right)\right):|f|<1\right\}$ and consider the transformation

$$
T: M_{k} \rightarrow M_{k}, f \mapsto \frac{1}{f}-\left[\frac{1}{f}\right] .
$$

For any $f \in \mathbb{F}_{k}\left(\left(x^{-1}\right)\right)$ we define a polynomial sequence $\left(a_{n}\right)_{n \geq 0}$ by

$$
a_{0}=[f] \quad \text { and, for } n \geq 1, \quad a_{n}=\left[\frac{1}{T^{n-1}(f-[f])}\right] .
$$

We easily check that

$$
f=a_{0}+\frac{1}{a_{1}+\frac{1}{a_{2}+\frac{1}{\ddots}}} .
$$

This expression is called the continued fraction expansion of $f$ and it will be simply denoted by $f=\left[a_{0}, a_{1}, a_{2}, \ldots\right]$. The sequence $\left(a_{i}\right)_{i \geq 0}$ is called the sequence of partial quotients of $f$. If it is bounded, we say that $f$ admits bounded continued fraction expansion.

Besides, we define two sequences of polynomials $\left(p_{n}\right)$ and $\left(q_{n}\right)$ by

$$
p_{0}=a_{0}, \quad q_{0}=1, \quad p_{1}=a_{0} a_{1}+1, \quad q_{1}=a_{1}
$$

and for any $n \geq 2 p_{n}=a_{n} p_{n-1}+p_{n-2}, \quad q_{n}=a_{n} q_{n-1}+q_{n-2}$.

By a straightforward computation, we can see that

$$
p_{n} q_{n-1}-p_{n-1} q_{n}=(-1)^{n-1} \text { and } \frac{p_{n}}{q_{n}}=\left[a_{0}, a_{1}, a_{2}, \ldots, a_{n}\right] .
$$

The sequence $\left(\frac{p_{n}}{q_{n}}\right)$ is called the sequence of convergent of $f$.

We will need the following two lemmas given by Baum and Sweet in [1]: 
Lemma 2.1. Consider $p, q \in \mathbb{F}_{2}[x]$ with $\operatorname{gcd}(p, q)=1$.

a. If $|q f-p|=2^{-(\gamma(q)+d)}, d>0$, then, there exists $n \in \mathbb{N}$ such that

$$
q=q_{n}, \quad p=p_{n} \quad \text { and } \quad \gamma\left(a_{n+1}\right)=d .
$$

b. If $|q f-p|=\frac{1}{|q|}$, then, there exists $n \in \mathbb{N}$ such that

$$
p=p_{n}+p_{n-1} \quad \text { and } \quad q=q_{n}+q_{n-1} .
$$

Lemma 2.2. Let $g_{1}, g_{2}, h_{1}, h_{2} \in \mathbb{F}_{2}[x]$ with $g_{1} h_{2}+g_{2} h_{1} \neq 0$. Then, any $f \in \mathbb{F}_{2}\left(\left(x^{-1}\right)\right)$ has bounded partial quotients if and only if $\frac{g_{1} f+h_{1}}{g_{2} f+h_{2}}$ has bounded partial quotients.

In 1976, Baum and Sweet gave in [1] some examples of formal series whose the sequence of partial quotients is unbounded. They have proved that the formal series $\left(\frac{P}{P+1}\right)^{\frac{1}{2^{n-1}}}$ admits unbounded partial quotients which are given explicitly.

\section{RESULTS}

Before giving the mains theorems, we introduce the following two lemmas.

Lemma 3.1. Let $P \in \mathbb{F}_{2}[x]$ such that the degree of $P$ is a multiple of 3 , then there is a unique $f \in \mathbb{F}_{2}\left(\left(x^{-1}\right)\right)$ such that $f^{3}=P$.

Proof. The uniqueness of $f$ is obvious. Let $P=x^{n}+a_{n-1} x^{n-1}+\cdots+a_{0}$ in which $n$ is a multiple of 3 . We seek $f=\sum_{i \geq n_{0}} f_{i} x^{-i}$ in $\mathbb{F}_{2}\left(\left(x^{-1}\right)\right)$ such that $f^{3}=P$ and $f_{n_{0}} \neq 0$. It is sufficient to determine the $f_{i}$ step by step. The equality $f^{3}=P$ yields first $n=-3 n_{0}$. Moreover, by identification, we get

$$
f_{n_{0}}=1, \quad f_{n_{0}+1}=a_{n-1}, \quad f_{n_{0}+2}=a_{n-2}-a_{n-1}^{2}, \quad f_{n_{0}+3}=a_{n-3}-a_{n-1}^{3}, \ldots
$$

Lemma 3.2. Let $P \in \mathbb{F}_{2}[x]$ such that $\gamma(P) \in 3 \mathbb{N}$. Then the polynomial $P$ is uniquely expressible as $P=S^{3}+T$, where $S, T \in \mathbb{F}_{2}[x]$ with $\gamma(T)<2 \gamma(S)$.

Proof. Write $P=x^{3 m}+a_{3 m-1} x^{3 m-1}+\cdots+a_{2 m} x^{2 m}+a_{2 m-1} x^{2 m-1}+\cdots+a_{0}$. From the equality $P=S^{3}+T$, together with the condition $\gamma(T)<2 \gamma(S)$, we deduce that $\gamma(S)=m$. Moreover, $S^{3}=x^{3 m}+a_{3 m-1} x^{3 m-1}+\cdots+a_{2 m} x^{2 m}+H$ where $\gamma(H)<2 k$. Thus, we write $S=\sum_{i=0}^{m} b_{i} x^{i} \in \mathbb{F}_{2}[x]$, then, as in the previous lemma, we compute recursively the coefficients $b_{m}, b_{m-1}, \ldots$ and then we get $P=S^{3}+T$ with $\gamma(T)<2 \gamma(S)$. 
Theorem 3.3. Let $P=S^{3}+T$ with $S, T \in \mathbb{F}_{2}[x]$, such that $T$ divides $P$ and $\gamma(T)<2 \gamma(S)$. Then the formal power series $g$ such that $g^{3}=P$ admits unbounded partial quotients.

Proof. We follows [1] fairly closely. Consider $f=\prod_{i \geq 0}\left(1+R^{-1}\right)^{4^{i}}$ where $R=T^{-1} P$. It is clear that $R \in \mathbb{F}_{2}[x]$ and $|R|>1$. We have

$$
\begin{aligned}
f^{3} & =\prod_{i \geq 0}\left(1+R^{-1}\right)^{3 \times 4^{i}}=\lim _{m \rightarrow+\infty}\left(1+R^{-1}\right)^{3 \times \sum_{i=0}^{m-1} 4^{i}}=\lim _{m \rightarrow+\infty}\left(1+R^{-1}\right)^{4^{m}-1} \\
& =\left(1+R^{-1}\right)^{-1} \lim _{m \rightarrow+\infty}\left(1+R^{-1}\right)^{4^{m}} .
\end{aligned}
$$

Since $\mathbb{F}_{2}\left(\left(x^{-1}\right)\right)$ is of characteristic 2 , we have then $f^{3}=\left(1+R^{-1}\right)^{-1}$ $\left(1+\lim _{m \rightarrow+\infty} R^{-4^{m}}\right)$. So, $f^{3}=\left(1+R^{-1}\right)^{-1}=\frac{1}{1+R^{-1}}=\frac{P}{P+T}$, then $f=$ $\left(\frac{P}{P+T}\right)^{\frac{1}{3}} \in \mathbb{F}_{2}\left(\left(x^{-1}\right)\right)$.

Let $d_{m}=\frac{\left(4^{m}-1\right)}{3}=\sum_{i=0}^{m-1} 4^{i}$. Then

$$
\begin{aligned}
(1+R)^{d_{m}} & =(1+R)^{\sum_{i=0}^{m-1} 4^{i}}=\prod_{i=0}^{m-1}(1+R)^{4^{i}} \\
& =\prod_{i=0}^{m-1} R^{4^{i}}\left(1+R^{-1}\right)^{4^{i}}=R^{d_{m}} \prod_{i=0}^{m-1}\left(1+R^{-1}\right)^{4^{i}}
\end{aligned}
$$

Thus, we get

$$
\begin{aligned}
R^{d_{m}} f & =R^{d_{m}} \prod_{i \geq 0}\left(1+R^{-1}\right)^{4^{i}}=R^{d_{m}} \prod_{0 \leq i<m}\left(1+R^{-1}\right)^{4^{i}} \prod_{i \geq m}\left(1+R^{-1}\right)^{4^{i}} \\
& =(1+R)^{d_{m}} \prod_{i \geq m}\left(1+R^{-1}\right)^{4^{i}}=(1+R)^{d_{m}}+(1+R)^{d_{m}}\left(R^{-1}\right)^{4^{m}}+K
\end{aligned}
$$

Therefore,

$$
R^{d_{m}} f-(1+R)^{d_{m}}=(1+R)^{d_{m}}\left(R^{-1}\right)^{4^{m}}+K .
$$

Moreover, we have $|K|<\left|(1+R)^{d_{m}}\left(R^{-1}\right)^{4^{m}}\right|=\left|R^{d_{m}}\left(R^{-1}\right)^{4^{m}}\right|$ since $\mid(1+$ $R)^{d_{m}}|=| R^{d_{m}} \mid$. So, we get

$$
\left|R^{d_{m}} f-(1+R)^{d_{m}}\right|=\left|R^{-\left(4^{m}-d_{m}\right)}\right|
$$


and then we have

$$
\left|R^{d_{m}} f-(1+R)^{d_{m}}\right|=2^{-\left(\gamma(R) d_{m}+\gamma(R) 4^{m}-2 \gamma(R) d_{m}\right)} .
$$

We have $\operatorname{gcd}\left(R^{d_{m}},(1+R)^{d_{m}}\right)=1$, moreover, $4^{m} \gamma(R)-2 \gamma(R) d_{m}>0$, then according to Lemma 2.1, there exists $i$ such that $\gamma\left(a_{i+1}\right)=4^{m} \gamma(R)-2 \gamma(R) d_{m}$. Obviously $\gamma\left(a_{i+1}\right)$ is unbounded, so, we conclude that the formal series $f=$ $\left(\frac{P}{P+T}\right)^{\frac{1}{3}}=\frac{1}{S}\left(S^{3}+T\right)^{\frac{1}{3}}$ admits unbounded partial quotients, but $S \in \mathbb{F}_{2}[x]$, then, by Lemma $2.2,\left(S^{3}+T\right)^{\frac{1}{3}}$ admits unbounded partial quotients.

Theorem 3.4. Let $S, T \in \mathbb{F}_{2}[x]$ such that $\gamma(T)<\gamma(S)$. Then the formal series $g$ such that $g^{3}=S^{3}+T$ admits unbounded partial quotients.

Proof. Let $P=S^{3}+T$. We begin the proof as in Theorem 3.3 until equality (3.1). Besides, multiplying (3.1) by $T^{d_{m}}$, we obtain

$$
\left|P^{d_{m}} f-(T+P)^{d_{m}}\right|=\left|T^{d_{m}}\left(T^{-1} P\right)^{-\left(4^{m}-d_{m}\right)}\right|=2^{-\left(\beta d_{m}+\left(\beta 4^{m}-2 \beta d_{m}-\alpha 4^{m}\right)\right)} .
$$

where $\beta=\gamma(P)$ and $\alpha=\gamma(T)$. We have to distinguish two cases:

(i) $\operatorname{gcd}(P, T)=1$ : since $\operatorname{deg} \gamma(T)<\frac{1}{3} \gamma(P)$ then $\beta 4^{m}-2 \beta d_{m}-\alpha 4^{m}>0$. Moreover, $\operatorname{gcd}\left(P^{d_{m}},(T+P)^{d_{m}}\right)=1$, then by Lemma 2.1 , there exists i such that $\gamma\left(a_{i+1}\right)=\beta 4^{m}-2 \beta d_{m}-\alpha 4^{m}$. It is clear that $\gamma\left(a_{i+1}\right)$ is unbounded.

(ii) $\operatorname{gcd}(P, T)=h \neq 1$ : there exists $P^{\prime}$ and $T^{\prime} \in \mathbb{F}_{2}[x]$ such that $P=h P^{\prime}$ and $T=h T^{\prime}$ with $\operatorname{gcd}\left(P^{\prime}, T^{\prime}\right)=1$. It is easy to verify that $\gamma\left(T^{\prime}\right)<\frac{1}{3} \gamma\left(P^{\prime}\right)$, and then the result is obvious by the first case.

So, in these two cases, $\left(\frac{P}{P+T}\right)^{\frac{1}{3}}$ admits unbounded partial quotients, then, as before, $\left(S^{3}+T\right)^{\frac{1}{3}}$ admits unbounded partial quotients .

Example 1. Let $f^{3}=S^{3}+1$ with $\gamma(S)>0$, then, by Theorem 3.3, the partial quotients of $f$ are unbounded.

Example 2. Let $f^{3}=S^{3}+x$ with $\gamma(S)>1$, then the partial quotients of $f$ are unbounded, by Theorem 3.3 if $S(0)=0$ and by Theorem 3.4 if $S(0) \neq 0$.

\section{ACKNOWLEDGMENTS}

We would like to thank Mabrouk Ben Ammar for reading the manuscript and helpful discussions. We are also grateful to the referee for his comments and suggestions. 


\section{REFERENCES}

1. L. E. Baum and H. M. Sweet, Continued fractions of algebraic power series in characteristitic 2, Ann. Math., 103 (1976), 593-610.

2. A. Khintchine, Continued fractions, Gosudarst. Isdat. Tecn.-Teor.Lit, MoscowLiningrad, 2nd ed., (1949), (MR $13 \neq 274 f$ ), in Russian.

3. A. Khintchine, Kettenbruche, B. G. Teubner, (1956), (MR $18 \neq 274 f)$.

4. W. H. Mills and D. P. Robbins, Continued fractions for certain algebraic power series, J. Number Theory, 23(3), (1986), 388-404.

5. D. P. Robbins, Cubic Laurent Series in Characteristic 2 with Bounded Partial Quotients, arXiv:math/9903092v1 [math.NT] 16 Mar. 1999.

K. Ayadi, M. Hbaib and F. Mahjoub

Département de Mathématiques

Faculté des Sciences de Sfax

BP 802, 3038 Sfax

Tunisie

E-mail: ayedikhalil@yahoo.fr mmmhbaib@gmail.com

faiza.mahjoub@yahoo.fr 